\title{
Comparison of two intraoral scanners based on three-dimensional surface analysis
}

\author{
Kyung-Min Lee(1)
}

\begin{abstract}
Background: This in vivo study evaluated the difference of two well-known intraoral scanners used in dentistry, namely iTero (Align Technology) and TRIOS (3Shape).

Methods: Thirty-two participants underwent intraoral scans with TRIOS and iTero scanners, as well as conventional alginate impressions. The scans obtained with the two intraoral scanners were compared with each other and were also compared with the corresponding model scans by means of three-dimensional surface analysis. The average differences between the two intraoral scans on the surfaces were evaluated by color-mapping. The average differences in the three-dimensional direction between each intraoral scans and its corresponding model scan were calculated at all points on the surfaces.
\end{abstract}

Results: The average differences between the two intraoral scanners were $0.057 \mathrm{~mm}$ at the maxilla and $0.069 \mathrm{~mm}$ at the mandible. Color histograms showed that local deviations between the two scanners occurred in the posterior area. As for difference in the three-dimensional direction, there was no statistically significant difference between two scanners.

Conclusions: Although there were some deviations in visible inspection, there was no statistical significance between the two intraoral scanners.

Keywords: Intraoral scan, Three-dimensional surface analysis, Digital impression

\section{Background}

With the advances in computer technology, digital dental models are now being widely used for orthodontic diagnosis and treatment planning. The use of digital models alleviates many of the challenges posed by plaster models made from conventional impressions, which include the burden of storage, the risk of damage or breakage, and the difficulties in sharing the data with other clinicians involved in the patients' care $[1,2]$. Digital dental models can be created through either indirect or direct techniques. Indirect methods involve laser scanning or computed tomographic imaging of the alginate impressions or plaster models, and direct methods involve intraoral scanners. With the introduction of chairside intraoral scanners, interest in obtaining

Correspondence: ortholkm@jnu.ac.kr

Department of Orthodontics, School of Dentistry, Chonnam National

University, 33 Yongbong-ro, Buk-gu, Gwangju 61186, South Korea digital dental model using the direct method has increased [3-5].

After the introduction of computer-aided design/computer-aided manufacturing (CAD/CAM) concepts into dental applications by Dr. Francois Duret at the Chicago Midwinter Meeting in 1989 [6, 7], several intraoral scanners have been introduced. Recently, a few intraoral scanners have been released on the market, including the iTero (Align Technologies), TRIOS (3Shape), True Definition (3M ESPE), CEREC Omnicam (Sirona), and CS 3600 (Carestream Dental) [8, 9].

The accuracy of intraoral scanners has been evaluated for both single abutment [10-13] and short-span fixed dental prostheses [14-16]. To determine the accuracy of intraoral scanners, researchers have performed in vitro studies using reference models [17-20]. Although shortspan intraoral scans have exhibited excellent accuracy, few in vivo studies have investigated the accuracy of 
intraoral scans on whole dentition in the clinical setting. Kuhr et al. [21] developed a new method of measuring the trueness of full-arch intraoral scans using reference spheres. However, the authors investigated only lower teeth [21]. Anh et al. [22] compared the precision of images acquired using the iTero and TRIOS intraoral scanners; however, they performed in vitro studies using fabricated dental arch models.

The iTero and TRIOS scanners allow full-arch scanning and do not require powdering of the tooth surfaces. Moreover, these two scanners have incorporated the orthodontic application software within the scanners. To our knowledge, iTero and TRIOS are the two bestknown commercially available intraoral scanners in dentistry; therefore, it is important to compare their relative scanning accuracy (Table 1). We cannot assume that all intraoral scanners will produce the same level of clinically acceptable results, and it would be beneficial to see how the measurements compare between different scanners. With this in mind, the present study aimed to compare the scanning results of the iTero and TRIOS scanners.

\section{Methods}

Thirty-two participants were enrolled in the study after providing informed consent. This study was approved by the Institutional Review Board for Medical Science at the Chonnam National University Hospital, Gwangju, Korea (CNUDH-2015-003). The inclusion criteria were complete permanent dentition, with no missing tooth and no crown or bridge restoration. In addition, participants with moderate or severe crowding and dentofacial deformity were excluded from this study.

\section{Intraoral scanning with TRIOS and iTero}

Intraoral scans of 32 participants were included in the present study. Each participant underwent intraoral scanning with TRIOS (3Shape, Copenhagen, Denmark) and iTero (version 4.0; Align Technology, San Jose, CA), as well as alginate impression. All intraoral scans with the iTero and TRIOS scanners were recorded by a single examiner. The scanners were calibrated every 8 days according to the manufacturers' recommendation. TRIOS scanning was performed following the instructions of the manufacturer. In brief, the scanning was started from the left side and continued to the right side along the occlusion. After the occlusal surfaces were scanned, lingual and buccal surface scans were performed. In the upper arch, the occlusal surfaces were scanned first, in the same manner as in the lower arch, whereas the buccal and lingual surfaces were scanned in order. When scanning the occlusal surfaces, the scanner head was kept at $0-5 \mathrm{~mm}$ from the tooth. For the scanning of the buccal and lingual surfaces, the scanner tip was rolled $45^{\circ}-90^{\circ}$ to the buccal and lingual sides, respectively. The image could be continuously viewed on a screen during the scanning process, which allowed direct visual feedback to ensure that no areas were missed. After scanning, all scan data were sent to the OrthoAnalyzer ${ }^{\mathrm{m}}$ (3Shape, Copenhagen, Denmark) software program, where they were reprocessed as a stereolithography (STL) file. The iTero scanning was performed in a predetermined sequence. The mandibular left area was scanned from the second molar to the incisor. The mandibular right area was scanned from the second molar. In the maxilla, the right quadrant was scanned first. The scan data were reprocessed as an STL file.

\section{Conventional alginate impression taking}

Alginate impressions (Cavex Normal set; Cavex Holland BV, Haarlem, The Netherlands) for the maxilla and mandible were taken in a metal tray. Each impression was rinsed with tap water and disinfected via spraying (CONTINU Dental Impression Disinfectant, Premium Plus, Bournemouth, UK). After disinfection, the impression was directly poured with dental stone. The stone casts were stored for $5-7$ days at a temperature of $23{ }^{\circ} \mathrm{C}$ $\pm 1{ }^{\circ} \mathrm{C}$ and humidity of $40 \% \pm 10 \%$. The dental models were scanned with a laboratory scanner (Orapix, Seoul, Korea). By means of a reverse engineering software program (Rapidform 2006; 3D Systems, Rock Hill, SC), the laboratory-scanned file was converted to STL format. The adjacent gingival tissue was deleted along the margin of the clinical crown to allow accurate best-fit alignment of the crowns.

\section{Comparison of the two intraoral scanners based on three- dimensional surface analysis}

The scanning results were compared by using threedimensional analysis with "shell/shell deviation" software commands regarding the average surface differences with subsequent color-coded charts. Two intraoral scans were registered by using the software's best-fit algorithm, and overall, three-dimensional comparisons were performed. Since the presence of adjacent soft tissue could increase the range of error, these areas were deleted,

Table 1 Two commercially available intraoral scanners used in this study

\begin{tabular}{llll}
\hline Scanner & Manufacturer & Software application & Scanning technology \\
\hline iTero & Align Technology & iOC & Parallel confocal microscopy \\
TRIOS & 3Shape & OrthoAnalyzer & Confocal microscopy \\
\hline
\end{tabular}



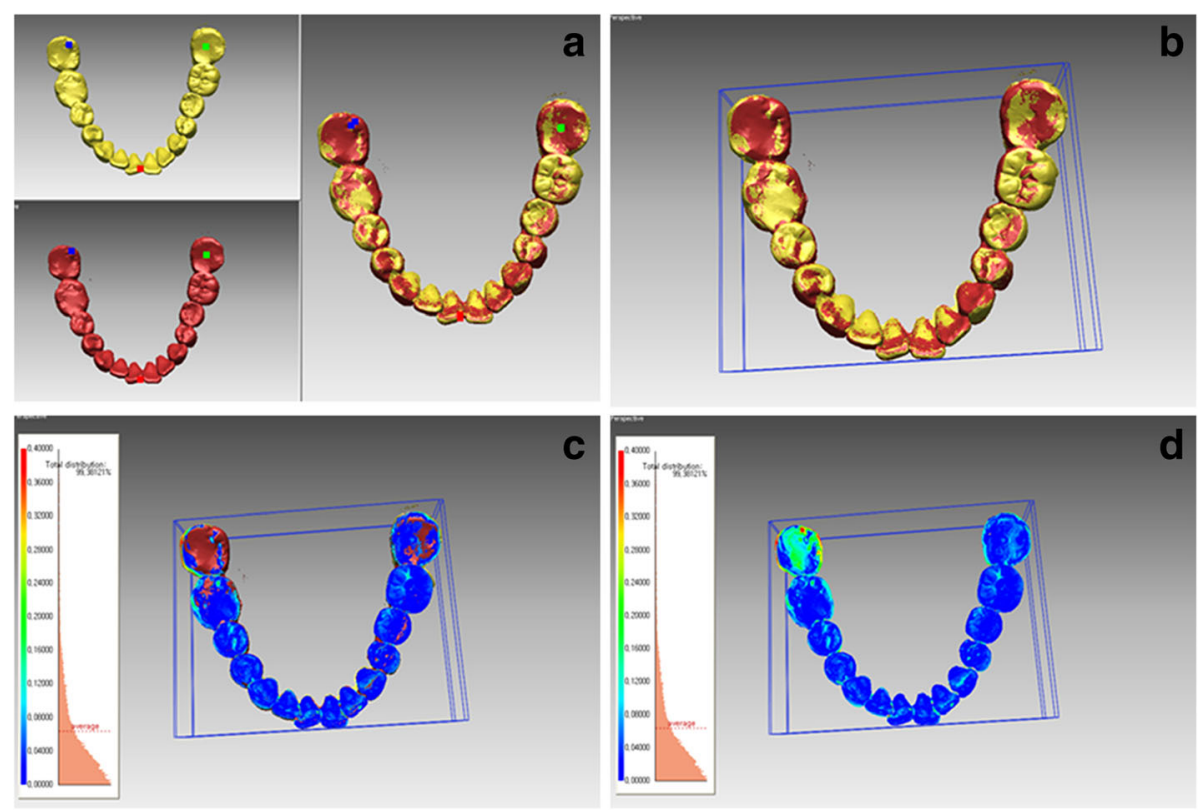

Fig. 1 Registration of the three-dimensional models created by iTero (yellow) and TRIOS (red). a Initial registration by three corresponding points on each of the two models. b Automatic fine registration for final registration. c Computation of average deviations between the two intraoral scans at all points on the surfaces. $\mathbf{d}$ Color-coded charts for qualitative evaluation

along with the gingival margin, to allow superimposition of the clinical crowns. The initial registration involved the selection of three corresponding points on each of the two intraoral scans. Subsequently, automatic fine registration was used to finalize the registration (Fig. 1). The average deviations between the two intraoral scans at all points on the surfaces were computed by using the "shell/shell deviation" function in the program. To remove outlier values, the calculation tolerance value in the program was set at $1.0 \mathrm{~mm}$ [23]. In addition, the differences between the two scans were evaluated by means of color histograms.
Deviations in the three-dimensional direction were also calculated in the incisor and molar regions. Three reference points were selected, and the point-to-point distance between each point on the intraoral scan and the corresponding laser-scanned model was computed. As for the reference points, the midpoint between the central incisors and mesiolingual cusp tip of the right and left first molars was determined. The point-to-point distance was regarded as the displacement of the intraoral scan to the laser-scanned model, and the relative distance between each intraoral scan and laserscanned model was calculated. In addition, the means

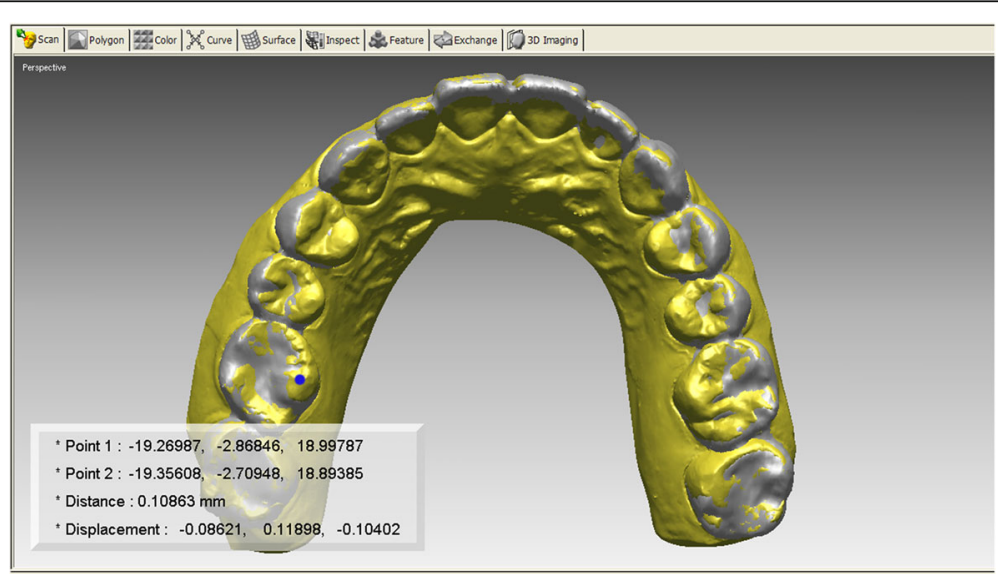

Fig. 2 Discrepancies in the three-dimensional direction were calculated in the incisor and molar regions. Three reference points were selected, and the point-to-point distance between each point on the intraoral scan and the corresponding model scan was computed. The point-to-point distance was regarded as the displacement of the intraoral scan to the model scan, and the relative distance was calculated 
Table 2 Average deviations ( $\mathrm{mm}$ ) between the iTero and TRIOS scanners obtained from a three-dimensional superimposition

\begin{tabular}{lll}
\hline & \multicolumn{2}{l}{ Average deviations } \\
\cline { 2 - 3 } & Mean & SD \\
\hline Maxilla & 0.057 & 0.018 \\
Mandible & 0.069 & 0.012 \\
\hline
\end{tabular}

and standard deviations were computed for each $X-, Y_{-}$, and $Z$-coordinate direction to evaluate which direction of the discrepancy contributed to the degree of overall discrepancies (Fig. 2). In order to analyze the differences between the two intraoral scanners, the paired $t$ test was used to compare the values using SPSS software package (version 23.0; SPSS Inc., Chicago, IL).

\section{Results}

The average deviations between the two intraoral scans were $0.057 \mathrm{~mm}$ in the maxilla and $0.069 \mathrm{~mm}$ in the mandible (Table 2). The color histogram showed that local deviations between the two scanners occurred in the posterior area (Fig. 3). In the three-dimensional deviations, the intraoral scans presented a minor displacement to the models; however, there were no statistically significant differences between the two scanners (Table 3).

\section{Discussion}

The average deviations between the two intraoral scanners were within $0.07 \mathrm{~mm}$. There is no clear consensus regarding the clinically or medico-legally acceptable amount of error in clinical orthodontics. Many researchers have suggested what they consider to be a clinically significant difference. Hirogaki et al. [24] suggested that orthodontic study models' accuracy should be about $0.30 \mathrm{~mm}$, while Schirmer and Wiltshire [25] reported that a measurement difference of less than $0.20 \mathrm{~mm}$ was clinically acceptable and Bell et al. [26] suggested that a measurement difference within $0.27 \mathrm{~mm}$ was clinically insignificant. With regard to the previous studies on the clinical acceptability in plaster models, the differences between two scanners of less than $0.07 \mathrm{~mm}$ in our results indicate that both intraoral scanners can be used in clinical orthodontics. Vasudavan et al. [27] compared intraoral scanning and conventional impression techniques for fabrication of orthodontic retainers. The clinical acceptability of retainers did not differ significantly by fabrication method, and the authors concluded that digital scans were considered acceptable [27]. Interestingly, the retainers made from digital scans were preferred significantly more often by the orthodontist than those made from alginate impressions.

In the present study, errors in the plaster model manufacturing process should be taken into consideration. Although alginate impression has potential errors, it is still being used for fabricating a diagnostic model. As plaster model and intraoral scan are used together in the clinics, it is necessary to evaluate the agreement between alginate impression and intraoral scanner.

Confocal laser scanning microscopy is used for intraoral scanner systems [28]. This technique is used to

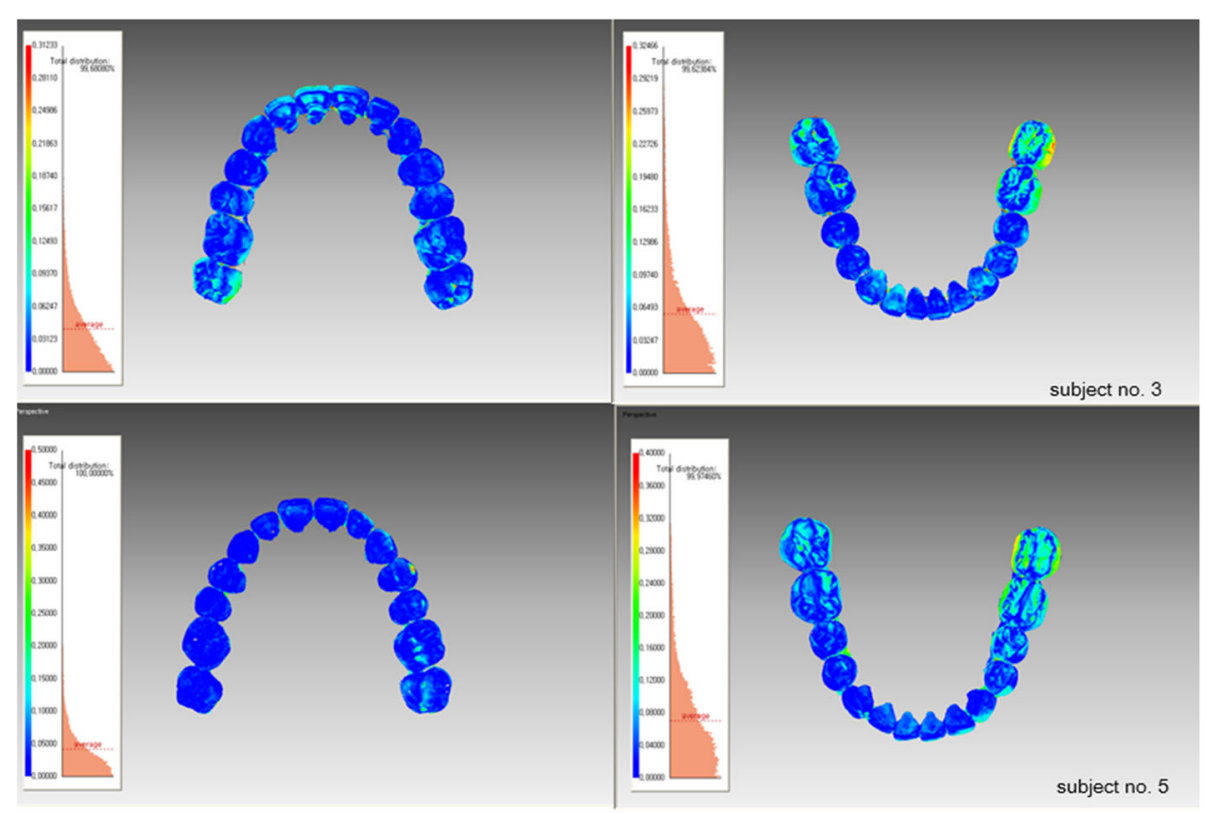

Fig. 3 Color-coded charts of the scanning results between the iTero and TRIOS scanners. The two images above are the scans of subject no. 3, and the two images below are the scans of subject no. 5. Discrepancies occurred in the posterior areas, particularly in the mandible on both cases 
Table 3 Three-dimensional discrepancy between each intraoral scan and model in the incisor and molar regions and its comparison between the iTero and TRIOS scanners (unit: $\mathrm{mm}$ )

\begin{tabular}{|c|c|c|c|}
\hline & iTero vs model scan & TRIOS vs model scan & \\
\hline & Mean \pm SD & Mean \pm SD & \\
\hline \multicolumn{4}{|l|}{ Maxilla } \\
\hline \multicolumn{4}{|c|}{ Midpoint between central incisors ${ }^{*}$} \\
\hline X-directional displacement & $0.06 \pm 0.04$ & $0.04 \pm 0.03$ & 0.311 \\
\hline Y-directional displacement & $0.03 \pm 0.10$ & $0.02 \pm 0.10$ & 0.758 \\
\hline Z-directional displacement & $0.01 \pm 0.06$ & $0.01 \pm 0.09$ & 0.508 \\
\hline \multicolumn{4}{|c|}{ First molar mesiolingual cusp, right ${ }^{\dagger}$} \\
\hline X-directional displacement & $0.11 \pm 0.09$ & $0.08 \pm 0.09$ & 0.738 \\
\hline Y-directional displacement & $-0.11 \pm 0.07$ & $-0.09 \pm 0.08$ & 0.386 \\
\hline Z-directional displacement & $0.04 \pm 0.08$ & $0.02 \pm 0.11$ & 0.672 \\
\hline \multicolumn{4}{|c|}{ First molar mesiolingual cusp, left ${ }^{\ddagger}$} \\
\hline X-directional displacement & $-0.11 \pm 0.11$ & $-0.06 \pm 0.12$ & 0.377 \\
\hline Y-directional displacement & $-0.01 \pm 0.10$ & $-0.06 \pm 0.07$ & 0.281 \\
\hline Z-directional displacement & $0.04 \pm 0.08$ & $0.05 \pm 0.04$ & 0.838 \\
\hline \multicolumn{4}{|l|}{ Mandible } \\
\hline \multicolumn{4}{|c|}{ Midpoint between central incisors ${ }^{\S}$} \\
\hline X-directional displacement & $-0.02 \pm 0.06$ & $0.02 \pm 0.08$ & 0.168 \\
\hline Y-directional displacement & $-0.07 \pm 0.13$ & $-0.09 \pm 0.09$ & 0.757 \\
\hline Z-directional displacement & $-0.02 \pm 0.09$ & $-0.09 \pm 0.10$ & 0.069 \\
\hline \multicolumn{4}{|c|}{ First molar mesiolingual cusp, right" } \\
\hline X-directional displacement & $0.01 \pm 0.12$ & $0.07 \pm 0.10$ & 0.163 \\
\hline Y-directional displacement & $0.12 \pm 0.11$ & $0.12 \pm 0.08$ & 0.866 \\
\hline Z-directional displacement & $0.09 \pm 0.17$ & $0.05 \pm 0.11$ & 0.410 \\
\hline \multicolumn{4}{|c|}{ First molar mesiolingual cusp, left" } \\
\hline X-directional displacement & $0.01 \pm 0.11$ & $0.02 \pm 0.12$ & 0.382 \\
\hline Y-directional displacement & $0.07 \pm 0.11$ & $0.10 \pm 0.06$ & 0.539 \\
\hline Z-directional displacement & $0.15 \pm 0.17$ & $0.15 \pm 0.07$ & 0.964 \\
\hline
\end{tabular}

$P$ values were obtained from paired $t$ test. $X, Y$, and $Z$ directions indicate mediolateral, superoinferior, and anteroposterior directions, respectively.

$S D$ standard deviation

*In the maxilla, positive value in the $X, Y$, and $Z$ directions indicates medial, apical, and anterior displacement

${ }^{\dagger}$ In the maxilla, negative value in the $X, Y$, and $Z$ directions indicates buccal, occlusal, and posterior displacement

${ }^{\ddagger}$ In the maxilla, negative value in the $X, Y$, and $Z$ directions indicates palatal, occlusal, and posterior displacement

${ }^{\S}$ In the mandible, negative value in the $X, Y$, and $Z$ directions indicates lateral, apical, and lingual displacement

"In the mandible, positive value in the $X, Y$, and $Z$ directions indicates lingual, occlusal, and anterior displacement

"In the mandible, positive value in the $X, Y$, and $Z$ directions indicates buccal, occlusal, and anterior displacement

acquire in-focus images from selected depths, a process known as optical sectioning (high-resolution optical images with depth selectivity) [29]. The iTero scanner employs a parallel confocal imaging technique with an array of incident red laser beams [30]. It is important to maintain the scanning wand at a certain focal distance while scanning. The TRIOS intraoral system also works according to the principle of confocal microscopy, with a fast scanning time. A fundamental characteristic of the TRIOS system is the variation of the focal plane without moving the scanner toward the subject being scanned [30]. The TRIOS system has the feature of telecentricity in the space of the subject being scanned, and it is possible to shift the focal plane while keeping telecentricity and magnification ratio [30]. We evaluated the light wave of the iTero and TRIOS scanners using polarization beam splitter and quarter-wave plate (Thorlabs Inc., Newton, NJ). It was found that the light of the TRIOS scanner was polarized, whereas the light of the iTero scanner was not polarized. The TRIOS scanner was found to use linear-to-circular polarization by the quarter-wave plate. A wave plate is an optical device that alters the polarization state of a light wave traveling through it. Two common types of wave plates are the half-wave plate, which shifts the polarization direction of linearly polarized light, and the quarter-wave plate, 
which converts linearly polarized light into circularly polarized light and vice versa [31]. In consideration of the polarization system of the TRIOS scanner, which might block the scattered reflections, this scanner might have better optical performance than iTero.

In addition, the iTero and TRIOS systems both capture single images of each tooth and produce an assembled virtual model of the whole dentition. This stitching process might produce systematic errors; however, because the stitching algorithms of the iTero and TRIOS scanners are not known, their contribution to such errors cannot be explained. Intraoral conditions such as saliva, breathing, movement of the tongue, and limited oral space can also contribute to scanning inaccuracies. For example, it is difficult for the scanner tip to access the lower posterior areas, owing to tongue movement and limited mouth opening. Flügge et al. [32] found that intraoral scanning was less precise than extraoral model scanning, indicating that the intraoral conditions contribute to the inaccuracy of scans. The accuracy can also be affected by the examiner's technical skill at intraoral scanning. To avoid such bias, in the present study, intraoral scans were obtained by the same examiner, who had experienced with over 100 cases of intraoral scanning. Long scanning times might induce errors in the stitching process of the captured images; the scanning times tend to decrease as the operator experience increased. In this study, the full-arch scan time for the iTero scanner was an average of $5 \mathrm{~min}$, while the TRIOS scanner was an average of $4 \mathrm{~min}$. However, further studies are needed to assess the scanning accuracy according to the clinician's experience.

There are few researches about the comparison of iTero and TRIOS scanner for in vivo and full-arch scan. For single abutment, the trueness and precision were higher in TRIOS than iTero scanner [33, 34]. Although Renne et al. [35] reported that trueness and precision for full-arch scanning was higher in iTero than in TRIOS, regarding scanning time, TRIOS was found to have the best balance of speed and accuracy [35]. Although there were no statistically significant differences between the two scanners in this study, some deviations might occur in the posterior areas, particularly in the mandible. In the clinical setting, the use of a careful and extended scanning protocol might improve the scanning results. With the continued development of digital impression technology, we will likely see the elimination of conventional impression taking in the near future.

\section{Conclusions}

Although there were some deviations in visible inspection, there was no statistical significance between the two intraoral scanners.

\section{Abbreviations}

CAD/CAM: Computer-aided design/computer-aided manufacturing;

STL: Stereolithography

\section{Acknowledgements}

Not applicable.

\section{Funding}

The present study was supported by Basic Science Research Program through the National Research Foundation of Korea (NRF) funded by the Ministry of Science, ICT and Future Planning (NRF-2014R1A1A1003559). This research was supported by Basic Science Research Program through the National Research Foundation of Korea (NRF) funded by the Ministry of Education (NRF-2017R1D1A1B03032132). All the scanners and materials used here belonged to the author, and nothing was provided by third-party or private companies.

\section{Availability of data and materials}

The STL files and the three-dimensional surface models obtained in this study with the two intraoral scanners belong to the author and are therefore available only upon request, after approval by the author.

\section{Ethics approval and consent to participate}

All participants provided informed consents. The present study was approved by the Chonnam National University Dental Hospital Institutional Review Board (CNUDH-2015-003).

\section{Consent for publication}

Not applicable.

\section{Competing interests}

The author declares that there are no competing interests.

\section{Publisher's Note}

Springer Nature remains neutral with regard to jurisdictional claims in published maps and institutional affiliations.

Received: 21 November 2017 Accepted: 23 January 2018

Published online: 12 February 2018

\section{References}

1. Christensen GJ. Will digital impressions eliminate the current problems with conventional impressions? J Am Dent Assoc. 2008;139:761-3.

2. Yuzbasioglu E, Kurt H, Turunc R, Bilir H. Comparison of digital and conventional impression techniques: evaluation of patients' perception, treatment comfort, effectiveness and clinical outcomes. BMC Oral Health. 2014;14:10.

3. Ender A, Mehl A. Full arch scans: conventional versus digital impressions-an in-vitro study. Int J Comput Dent. 2011;14:11-21.

4. van der Meer WJ, Andriessen FS, Wismeijer D, Ren Y. Application of intra-oral dental scanners in the digital workflow of implantology. PLoS One. 2012;7:e43312

5. Nayar S, Mahadevan R. A paradigm shift in the concept for making dental impressions. J Pharm Bioallied Sci. 2015;7(suppl 1):s213-5.

6. Duret F, Blouin JL. Optical impressions in the computer-assisted design and fabrication of dental crowns. J Dent Que. 1986;23:177-80.

7. Birnbaum NS, Aaronson HB, Stevens C, Cohen B. 3D digital scanners: a high-tech approach to more accurate dental impressions. Inside Dentistry. 2009;5:70-4.

8. Kachalia PR, Geissberger MJ. Dentistry a la carte: in-office CAD/CAM technology. J Calif Dent Assoc. 2010;38:323-30.

9. Focus on digital oral scanners. Orthodontic products. 2016:30-1. http:// www.orthodonticproductsonline.com.

10. Luthardt RG, Loos R, Quaas S. Accuracy of intraoral data acquisition in comparison to the conventional impression. Int J Comput Dent. 2005;8:283-94

11. Syrek A, Reich G, Ranftl D, Klein C, Cerny B, Brodesser J. Clinical evaluation of all-ceramic crowns fabricated from intraoral digital impressions based on the principle of active wavefront sampling. J Dent. 2010;38:553-9.

12. Lee SJ, Gallucci GO. Digital vs. conventional implant impressions: efficiency outcomes. Clin Oral Implants Res. 2013;24:111-5.

13. Seelbach P, Brueckel C, Wöstmann B. Accuracy of digital and conventiona impression techniques and workflow. Clin Oral Investig. 2013;17:1759-64. 
14. Giménez B, Özcan M, Martínez-Rus F, Pradíes G. Accuracy of a digital impression system based on parallel confocal laser technology for implants with consideration of operator experience and implant angulation and depth. Int J Oral Maxillofac Implants. 2014:29:853-62.

15. Giménez B, Özcan M, Martínez-Rus F, Pradíes G. Accuracy of a digital impression system based on active wavefront sampling technology for implants considering operator experience, implant angulation, and depth. Clin Implant Dent Relat Res. 2015;17(suppl 1):e54-64.

16. Papaspyridakos P, Gallucci GO, Chen CJ, Hanssen S, Naert I, Vandenberghe B. Digital versus conventional implant impressions for edentulous patients: accuracy outcomes. Clin Oral Implants Res. 2016;27:465-72.

17. Ender A, Mehl A. Accuracy of complete-arch dental impressions: a new method of measuring trueness and precision. J Prosthet Dent. 2013;109:121-8.

18. Patzelt SB, Vonau S, Stampf S, Att W. Assessing the feasibility and accuracy of digitizing edentulous jaws. J Am Dent Assoc. 2013;144:914-20.

19. Patzelt SB, Emmanouilidi A, Stampf S, Strub JR, Att W. Accuracy of full-arch scans using intraoral scanners. Clin Oral Investig. 2014;18:1687-94.

20. Ender A, Mehl A. In-vitro evaluation of the accuracy of conventional and digital methods of obtaining full-arch dental impressions. Quintessence Int. 2015;46:9-17.

21. Kuhr F, Schmidt A, Rehmann P, Wöstmann B. A new method for assessing the accuracy of full arch impressions in patients. J Dent. 2016;55:68-74.

22. Anh JW, Park JM, Chun YS, Kim M, Kim M. A comparison of the precision of three-dimensional images acquired by 2 digital intraoral scanners: effects of tooth irregularity and scanning direction. Korean J Orthod. 2016;46:3-12.

23. Noh H, Nabha W, Cho JH, Hwang HS. Registration accuracy in the integration of laser-scanned dental images into maxillofacial cone-beam computed tomography images. Am J Orthod Dentofac Orthop. 2011;140:585-91.

24. Hirogaki Y, Sohmura T, Satoh H, Takahashi J, Takada K. Complete 3-D reconstruction of dental cast shape using perceptual grouping. IEEE Trans Med Imaging. 2001;20:1093-101.

25. Schirmer UR, Wiltshire WA. Manual and computer-aided space analysis: a comparative study. Am J Orthod Dentofac Orthop. 1997;112:676-80

26. Bell A, Ayoub AF, Siebert P. Assessment of the accuracy of a threedimensional imaging system for archiving dental study models. J Orthod. 2003;30:219-23.

27. Vasudavan S, Sullivan SR, Sonis AL. Comparison of intraoral 3D scanning and conventional impressions for fabrication of orthodontic retainers. J Clin Orthod. 2010;44:495-7.

28. Koester CJ. A comparison of various optical sectioning methods. In: Pawley JB, editor. Handbook of biological confocal microscopy. 3rd ed. New York: Springer; 2006. p. 207-14

29. Babayoff $N$ and Glaser-Inbari I: Method and apparatus for imaging threedimensional structure. US Patent 2007/0109559; 2007.

30. Logozzo S, Zanetti EM, Franceschini G, Kilpelä A, Mäkynen A. Recent advances in dental optics-part I: 3D intraoral scanners for restorative dentistry. Opt Laser Eng. 2014;54:203-21.

31. Hecht E. Polarization. In: Hecht E, editor. Optics. 4th ed. Harlow: Pearson Education Limited; 2008. p. 327-86.

32. Flügge TV, Schlager S, Nelson K, Nahles S, Metzger MC. Precision of intraoral digital dental impressions with iTero and extraoral digitization with the iTero and a model scanner. Am J Orthod Dentofac Orthop. 2013;144:471-8.

33. Hack GD, Patzelt SBM. Evaluation of the accuracy of six intraoral scanning devices: an in-vitro investigation. ADA professional product review. 2015;10:1-5.

34. Imburgia M, Logozzo S, Hauschild U, Veronesi G, Mangano C, Mangano FG. Accuracy of four intraoral scanners in oral implantology: a comparative in vitro study. BMC Oral Health. 2017;17:92

35. Renne W, Ludlow M, Fryml J, Schurch Z, Mennito A, Kessler R, Lauer A. Evaluation of the accuracy of 7 digital scanners: an in vitro analysis based on 3-dimensional comparisons. J Prosthet Dent. 2017:118:36-42

\section{Submit your manuscript to a SpringerOpen ${ }^{\circ}$ journal and benefit from:}

- Convenient online submission

- Rigorous peer review

Open access: articles freely available online

- High visibility within the field

- Retaining the copyright to your article

Submit your next manuscript at $\boldsymbol{\nabla}$ springeropen.com 